\title{
Application of Digital Technology and PDA+APPS Mobile Terminal in Historical Architecture Culture Protection and Development
}

\author{
Zhi Feng LIU, Ying WANG, Dong Hui HUANG \\ Architectural Engineering Institute, Jin ling Institute of Technology, Nanjing, Jiangsu 210069, China
}

\begin{abstract}
In the wake of big data and Internet plus era, continuous infiltration of digital technology has been happening in various fields of social and economic development. As the most important material carrier of historical culture, the historical value of historical buildings is produced and accumulated in its historical evolution, and it can only be protected from being created again. Based on the background of digitization of cultural resources, this paper summarizes the relevant digital technologies for the digital translation of information on buildings of historical and cultural heritage, as a means to promote the spread of the PDA+APPS mobile terminal, so as to achieve the purpose of preservation, protection, management and publicity. Meanwhile, this paper analyzes the application of digital technology in this field and the prospect of its function.
\end{abstract}

\section{Research Background}

With the advent of big data and Internet plus era, continuous infiltration of digital technology has been happening in various fields of social and economic development, thus mutual cooperation among traditional industry and information industry is playing a key role in promoting the development of industrial transformation and upgrading. Historical buildings, as the most important material carrier of historical culture, the historical value of whom is produced and accumulated in its historical evolution, and can only be protected or developed protectively from being created again. Once lost, it will be lost forever.

Using digital technology to protect and develop historical architectural culture is a product of the development of information technology and computer technology. The relevant information of historical architecture culture heritage is digitally translated through computer network and software technology, by means of intelligent mobile terminals, and eventually achieve the purpose of preservation, protection and promotion[1].

The specific advantages are listed as follows:

(1) The digital acquisition and storage technology provides guarantee for the complete protection of historical architectural cultural resources, and the digital restoration and reproduction technology provides support for its effective inheritance;

(2) Virtual reality technology expands space for the development and utilization of these cultural resources;

(3) PDA+ APPS mobile terminal provides a wide publicity platform for the display and dissemination of the technology, broadening the horizon of the audience.

Compared with earlier studies of ancient books, dance and other intangible cultural resources, digital development and utilization of historic architecture is a relatively new topic. At present, those remaining historic buildings in the city, which are still in use and of sound quality, have a distinctive characteristic of the times and the local traditional culture. In addition, the use of new technology to preserve these precious intangible heritage information development and its utilization, will also become the inevitable trend in this field.

\section{Main Technical Means}

With the rapid advancement of computer and network technology, the depth and breadth of the research work about digital protection and development of historic architectural culture are also developing rapidly, and gradually formed an intensive, intelligent and systematic stage of technology on the basis of three-dimensional scanning technology, information modeling technology and virtual reality (VR).

PDA includes consumer goods and industrial grade, mainly refers to the intelligent mobile phone, tablet computer, POS machine, RFID reader and other handheld mobile intelligent terminal; APPS is an intelligent software rooted in certain operating system, known as the "application"(APP). PDA+APPS, which is based on Internet, equips those current mainstream like QQ, We Chat, micro-blog and other social network media on the intelligent mobile phone, tablet computer, portable computers and other mobile intelligent terminal

\footnotetext{
*Corresponding author: Email: wangying1212@jit.edu.cn
} 
for publicity and promotion.

The main technologies of digital protection and development of historical buildings include raw data acquisition and measurement technology, three-dimensional modeling technology and three-dimensional simulator technology.

\subsection{Raw Data Acquisition and Measurement Technology}

The raw data collection of historical buildings is the basis of digitization. The original data is usually obtained by field surveying, and its accuracy directly affects the accuracy of building rehabilitation design. The establishment of the database is to archive and protect the historical building information, and also to provide basis for the rehabilitation design.

In the past, people mainly used historical drawings, tables, text and other forms of recording to archive and preserve historical buildings, which cannot reflect the real effect of the building in addition to large quantity of data; While the use of photos, videos and other image archive is intuitive, it cannot bring about accurate physical data and structural relation. Thus, it is difficult to accurately protect those buildings or carry out scientific research, and the reconstruction, imitation and restoration work will be hard to access to. Therefore, GPS, total station, measurement robot and photogrammetry have been the representative of three-dimensional modern building surveying and mapping technology, which is not only gradually replacing the traditional two-dimensional measurement method but also improving and enriching the modern means of collecting original data. Among them, the more advanced digital mapping methods can be combined with $3 \mathrm{D}$ scanners to facilitate point sampling on different scales of real objects and obtain cloud data of different precision.

Through the processing, analysis and recognition algorithms of point cloud and grid, 3D surface model is constructed, and the $3 \mathrm{D}$ entity information is taken as the form of mapping results directly[2]. Compared with the traditional database which is made up of two-dimensional information, this kind of database which contains a lot of 3D building information is more comprehensive, intuitive and easy to check.

\subsection{Three-dimensional Modeling Technology}

On the basis of collecting original data through surveying and mapping, the two-dimensional data is transformed into 3D information model by using professional software. Modern computer technology has been able to overcome geographical restrictions to achieve real, rapid and accurate visual display, and can even interact with the environment model. In this way, it can not only reduce the man-made damage to the physical object, but also expand the publicity and bring economic benefits.
In addition to the three-dimensional scanning model mentioned above, the building modeling in the current industry is mainly based on the two-dimensional data obtained by surveying and mapping (such as space size, component size, graticule modeling photos, etc.), using universal professional modeling software (such as AutoCAD, 3DMax, Revit, SketchUp etc.), to establish certain three-dimensional modelt which can meet the requirements of surveying and mapping accuracy and scale representation. In the meantime, A large number of architectural models can form model libraries to provide protection for the construction of virtual simulation and preservation of architectural information.

\subsection{Three-dimensional Simulator Technology}

With the advancement of three-dimensional modeling technology, rendering technology and computer aided design (CAB) technology, three-dimensional simulator technology has become more and more widely used in various construction work. Through digital technology, professional tools are used to measure the plane and elevation of the existing historical buildings, then the original data are collected, processed, drawn and digitally modeled to form a digital resource library.

Through the intuitive image formed by digital software and media, virtual reality technology is used to reproduce the scene, and then realize the virtual simulation of historical buildings. The application of 3D digital technology is of great practical significance for the protection, renovation, data preservation, excavation of historical buildings and promotion of ancient architecture culture[3]. Among them, VR (Virtual Reality), also known as virtual environment (Virtual Environments), simulator technology (Simulator Technology) and so on, creates a 3D image world for the user in real-time to reflect the changes and interactions of the entities. In the realistic experience of seeing, listening, touching and smelling, the ordinary audience can explore and directly experience the historical building environment in the virtual, as if in the historical process of the building.

\section{Main Technical Applications}

At present, the digital protection and development of historic buildings include single building maintenance and renovation, data acquisition of historical blocks during their preservation and renovation, data analysis and processing, the effect of virtual display, forming an intensive resource sharing platform to facilitate the unified management of the government. In this way, the intelligent mobile terminal is used to spread the information, broadening the horizon of audience and improving data utilization level as well as the public awareness of protection. Figure 1 is a framework of digital technical protection and development of historical buildings. 


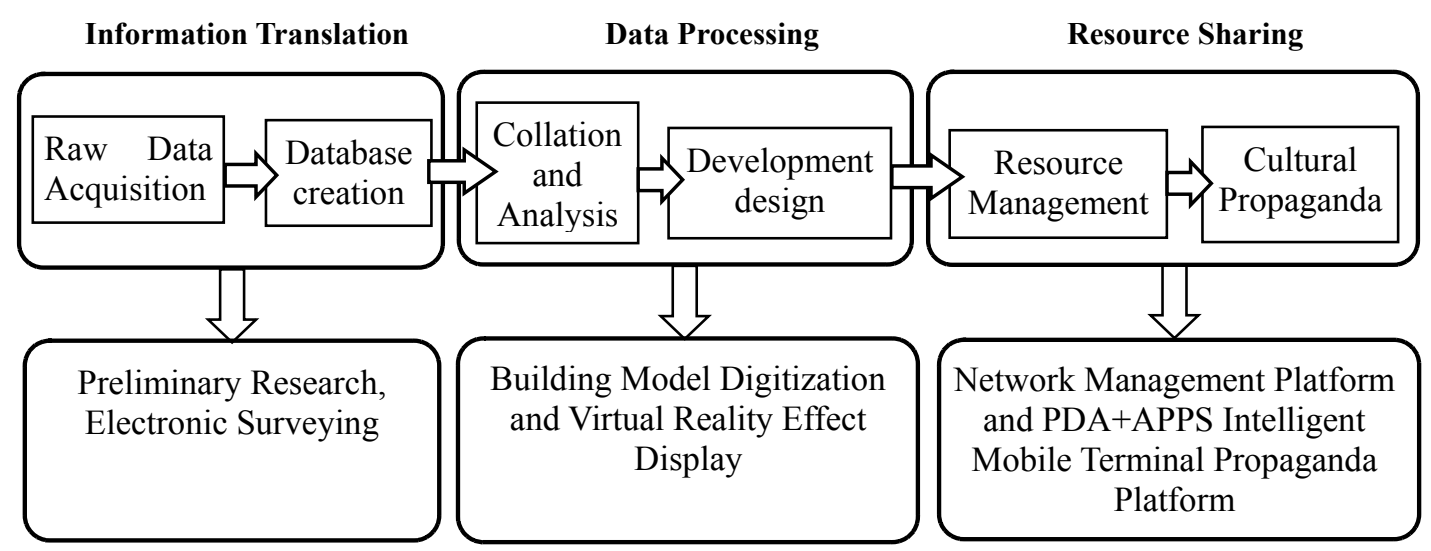

Figure 1 Framework of Digital Technical Protection and Development of Historical Buildings

\subsection{Protection and Renewal of Historical Buildings}

Under the background of rapid construction of modernization in the city, many historic buildings which are not included in the security unit have been frequently demolished or rebuilt, facing the danger of disappearing forever. Due to the complexity of the present situation, the protection and renewal of the historical buildings is also tend to be diversified. Especially for those badly damaged and badly preserved urban historical buildings, they should be restored at the site according to its original features. At the same time, the internal layout or structure should be properly reformed to meet the requirements of modern utilization[4].

Digital technology, on the one hand, is significant for the upcoming demolition, the overall relocation or rehabilitation of historical buildings, as detailed preservation of three-dimensional data will become an important basis for the restoration of its original shape. On the other hand, by means of digital technology and the accurate three-dimensional digital model, the engineering data, material data and process data of the historical buildings which have disappeared or are disappearing can be originally identified in the early stage of building information collection. In the meantime, the construction quality and feasibility of repair can be evaluated on this basis. Besides, the combination of digital 3D building model and virtual reality technology is also used for tourism development and cultural promotion.

\subsection{Preservation and Renovation of Historical Blocks}

As an important carrier of urban cultural heritage, historical blocks are also important elements of urban historical environment and characteristics of the times. Therefore, when it comes to the protection of cultural heritage, we should avoid the misunderstanding that only protection units instead of general houses and alleys should be paid attention to. We can never develop and construct our urban at the cost of losing historical blocks and urban traditional features. We should keep it firmly in mind that preservation is combined with utilization and development. With the advancement of digital technology, it plays an increasingly more important role in the whole renovation of historical blocks.

For example, located in the downtown of Nanjing, the modern historic district Meiyuan Village, who covers an area of about 45 thousand square meters, is one of the residential areas directly promoted by government during the period of Republic of China. From the perspective of construction features, in addition to the civilian housing built by the national government, there are also self-designed houses for middle and high class, and the forms of construction, land use area and living standards also show obvious diversification. Since Liberation, due to the complex ownership of property rights and other reasons, in those residential areas there still exist varying degrees of damage and other issues such as serious renovation and reconstruction, disunity of overall features and so on[5]. Recently, in order to meet the requirements of the cultural relics census and the protection and renovation works of historical blocks, a large-scale investigation has been carried out within the neighbourhood. In the course of the investigation, digital technology has also been applied in many places, mainly embodied in:

(1) By means of photogrammetry and three-dimensional measurements, relatively accurate architectural mapping data of nearly 60 modern houses within the neighbourhood was collected in the case of not or less disturbing local residents. Through AutoCAD, SketchUp and other model software, models that can reflect the shape, space, material of the building have been created as the basic data. At the same time, each residential monomer was numbered, a small database was formed based on location index, history status, elevation mapping, model data and other information, so as to provide effective resources for the overall style and environment improvement of the block .

(2) Classify and sort out the building information in the established database. On the one hand, according to the location, blocks was divided into three zones- the Meiyuan, Yongyuan and Taoyuan, where modern houses are more concentrated, so that it is beneficial to patch location management. On the other hand, those buildings 
were divided into three types: the western style villa, the detached house and the platoon apartment according to the architectural form and the living standard, and then we classified the existing modern houses separately. Through these methods, the system and retrieval of database are further strengthened.

(3) According to the current situation of blocks in the database, a unified renovation scheme was formulated for most of those modern residence (especially residence along the street). Through digital software and media, an intuitive image was formed, and a static display of the restored part, the facade shape, the exterior wall material and the color of the restored single building was made .The use of 3D simulation technology makes people feel personally on the scene of the effect of renovation, and certain publicity was made through the network platform.

\subsection{Create a platform for digital management and publicity}

At present, in the process of digital construction of national cultural resources, low level construction and low sharing degree are common. Therefore, it is necessary to establish an effective resource management and publicity sharing platform through effectively organizing and standardizing the description of resources. Digital management and publicity platform mainly refers to the implementation of information integrated management and publicity system based on the established database by means of Web and other network technologies. Through this platform, the relevant written materials, pictures, multimedia and video of the historical buildings in the database can be digitally processed and edited, and many types of mainstream social network media is equipped with a variety of mobile terminal in the form of "PDA+APPS". In this way, the intensive means of promotion as well as the sharing of historic buildings resources can be realized.

Through the creation of digital platform, first of all, it is beneficial for relevant departments to improve the management of historical buildings and ensure the current management more rigorous and efficient; Secondly, the network sharing of digital cultural resources also provides technical support for the protection and development of historical buildings. In addition, the digital platform in the future might help the government realize the information management of historic buildings's preservation and redevelopment, including the implementation of relevant policies and regulations, broadening the investment channels and other functions.

For example, the scope of platform management can involve government, developers, masses, historical construction operators and historical construction managers an so on Among them, the government can publish policies through the platform, mainly focuses on management and guidance. Owners of property rights, managers and social masses can also obtain all the information about historical buildings in a timely manner through this platform, which can provide services for tourism development, cultural exchanges and educational research,etc. Ordinary users are also end-users, they can search, check, download certain information about historical building resources as well as participate in the feedback of these resources object[6].

\section{Conclusion and Outlook}

At present, in some specific domestic cases where the digital development of historic buildings are mainly concentrated in , there are still lacks in established system and uniform standards. Besides, the integrity and accuracy of the database, the innovative application of its digital function still need further study. At the same time, the digital resources of historical buildings are mostly limited to the internal sharing of the research institutions, and have not been effectively developed and exchanged in a wider scope. Therefore, the digital technology and PDA+APPS mobile terminal is a creative combination of modern information technology and mobile communication technology, it can effectively translate, preserve and protect the data of historical architecture culture. And through digital management and publicity platform it is feasible to realize intensive management, utilization, publicity and sharing. It is believed that with the advancement of technology and the continuous improvement of policies and measures, digital development will become a new starting point for the protection and cultural heritage of modern historical buildings.

\section{References}

1. Zhang Hui, et. Extended research on the function of digital application of public cultural resources [J]. information and communication, 2014.10 (142): 78-79.

2. Fan Zhang Wei, Xing Yu. Research on Ancient Architecture Conservation Based on Digital Technology [J]. Beijing surveying and mapping, 2010 (3): 18-21.

3. Wang Haiying, Liu Zhifeng. Conservation and Reuse of Digital Technology in the Minguo Architectural Landscape of Culture $[\mathrm{J}]$. architecture and culture, 2015.08 (137): 113-114.

4. Wang Ying, Wang Yongping. Protection and Renovation of Historical Architecture in Block Reconstruction: Taking Two Republican - Style Buildings in Shengzhou Road in Nanjing [J]. Hundred Schools in Arts, 2012, 28 (8): 148-152.

5. Wang Ying, Wang Yongping. A Preliminary Exploration on the Residential Area Built Before 1949 in Meiyuanxincun, Nanjing [J]. Hundred Schools in Arts, 2009 (04): 39-45.

6. Li Yan. Suggestionon the Development of Digitization Management Platform for Haikou Historic Architecture [J]. Journal of Hainan University, Natural Science Edition,.2009.6 (27): 173-175. 